\title{
Application of the mid-IR radio correlation to the G sample and the search for advanced extraterrestrial civilisations ${ }^{\star}$
}

\author{
M. A. Garrett ${ }^{1,2}$ \\ 1 ASTRON, Netherlands Institute for Radio Astronomy, Postbox 2, 7990 AA Dwingeloo, The Netherlands \\ e-mail: garrett@astron.nl \\ 2 Leiden Observatory, Leiden University, PO Box 9513, 2300 RA Leiden, The Netherlands
}

Received 7 June 2015 / Accepted 11 August 2015

\begin{abstract}
Wright et al. (2014, ApJ, 792, 26) have embarked on a search for advanced Karadashev Type III civilisations via the compilation of a sample of sources with extreme mid-IR emission and colours. The aim is to furnish a list of candidate galaxies that might harbour an advanced Kardashev Type III civilisation; in this scenario, the mid-IR emission is then primarily associated with waste heat energy byproducts. I apply the mid-IR radio correlation to this Glimpsing Heat from Alien Technology ( $\hat{\mathrm{G}})$ sample, a catalogue of 93 candidate galaxies compiled by Griffith et al. (2015, ApJS, 217, 25). I demonstrate that the mid-IR and radio luminosities are correlated for the sample, determining a k-corrected value of $q_{22}=1.35 \pm 0.42$. By comparison, a similar measurement for 124 galaxies drawn from the First Look Survey (FLS) has $q_{22}=0.87 \pm 0.27$. The statistically significant difference of the mean value of $q_{22}$ for these two samples, taken together with their more comparable far-IR properties, suggests that the G sample shows excessive emission in the mid-IR. The fact that the $\hat{\mathrm{G}}$ sample largely follows the mid-IR radio correlation strongly suggests that the vast majority of these sources are associated with galaxies in which natural astrophysical processes are dominant. This simple application of the mid-IR radio correlation can substantially reduce the number of false positives in the $\hat{G}$ catalogue since galaxies occupied by advanced Kardashev Type III civilisations would be expected to exhibit very high values of $q$. I identify nine outliers in the sample with $q_{22}>2$ of which at least three have properties that are relatively well explained via standard astrophysical interpretations e.g. dust emission associated with nascent star formation and/or nuclear activity from a heavily obscured AGN. The other outliers have not been studied in any great detail, and are deserving of further observation. I also note that the comparison of resolved mid-IR and radio images of galaxies on sub-galactic $(\mathrm{kpc})$ scales can also be useful in identifying and recognising artificial mid-IR emission from less advanced intermediate Type II/III civilisations. Nevertheless, from the bulk properties of the Ĝ sample, I conclude that Kardashev Type III civilisations are either very rare or do not exist in the local Universe.
\end{abstract}

Key words. astrobiology - galaxies: general - galaxies: star formation - radio continuum: galaxies - infrared: general

\section{Introduction}

In a series of papers, Wright et al. (2014a) and Wright et al. (2014b) have presented a detailed description of our current ability to detect the signature of advanced Kardashev Type III civilisations (Kardashev 1964) via the prominent waste heat signature they are expected to produce. Type III civilisations are defined by Kardashev (1964) as those capable of harnessing the stellar energy supply of a galaxy ( $\sim 10^{38}$ Watts). Indeed previous studies (e.g. Carrigan 2009), suggest that constructs such as Dyson spheres (Dyson 1960) will radiate most of their waste heat energy at mid-IR wavelengths, corresponding to temperatures of $\sim 100-600 \mathrm{~K}$

Wright et al. have embarked on a novel project called Glimpsing Heat from Alien Technology $(\hat{G})$ based on the results of an all-sky mid-IR survey conducted by the WISE mission (Wright et al. 2010). In particular, Griffith et al. (2015) have recently produced a list of 93 sources (from an original sample of 100000 resolved WISE detections) that exhibit both extreme mid-IR emission and mid-IR colours. If the radiation measured by WISE is interpreted as waste heat emission from an advanced civilisation, the source sample includes galaxies

* Table 1 is available in electronic form at http://www . aanda.org reprocessing more than $25 \%$ of their starlight into the mid-IR (i.e. $\gamma>0.25$ in the formalism of Wright et al. 2014b). While some of these sources are well known (e.g. Arp 220), the majority have not been individually studied in any great detail. One significant problem with the $\hat{G}$ approach is the large number of false positives expected in the sample; in particular, there are many ways in which emission in the mid-IR can arise via natural astrophysical processes, e.g. the reprocessing of starlight or active galactic nucleus (AGN) radiation by dust.

One way of identifying bona fide Type III civilisations is to identify outliers in well-determined scaling laws for galaxies, e.g. the Tully-Fisher relation Annis (1999). I argue here, that the infrared radio correlation can also be used in a similar way. The original infrared radio correlation is a fundamental relation for galaxies (van der kruit 1971; Helou et al. 1985; Condon 1992; Yun et al. 2001), covering at least 5 orders of magnitude in luminosity, holding over a wide range of different redshifts, and extending well into the far-IR/mid-IR, and submillimetre domains (Carilli \& Yun 1999; Garrett 2002; Elbaz et al. 2002; Ivison et al. 2002; Appleton et al. 2004). Studies of the correlation usually quote $q$, the logarithm of the ratio of the IR to radio flux densities (luminosities), the latter typically being measured at $1.4 \mathrm{GHz}$ $(\lambda 20 \mathrm{~cm}) . \mathrm{K}$-corrected values of $q$ vary from $\sim 2.3$ in the far-IR 
(60-100 micron) to $\sim 1$ in the mid-IR ( 24 micron), and reflect the evolvution of the bolometric spectral energy distribution (SED) of a galaxy across the radio, near-IR, mid-IR, and far-IR domains. The correlation is strongest for star forming galaxies, but also applies to many other galaxy types, including radio quiet AGNs (Roy et al. 1998).

The physical explanation for the strength of the correlation is that both the non-thermal radio emission and the thermal IR emission are related to mechanisms driven by massive star formation. For galaxies in which the bulk of the mid-IR emission is associated with waste heat processes, there is no obvious reason why artifical radio emission would be similarly enhanced. While the continuum radio emission level might increase through the use of advanced communication systems, the amount of waste energy deposited in the radio domain is likely to be many orders of magnitude smaller than that expected at mid-IR wavelengths. As a consequence, I propose to apply the mid-IR radio correlation to the $93 \hat{\mathrm{G}}$ sources presented in Table 9 of Griffith et al. 2015. In particular, galaxies that are associated with Type III civilisations should appear as outliers in the midIR radio correlation with extremely high values of $q$. It should be noted that this deviation is opposite in sense to other frequent outliers, i.e. radio-loud AGN with systematically low values of $q$.

In this paper, I calculate values of $q$ for the $\hat{G}$ sample, identifying interesting outliers that are deserving of further study. In Sect. 2 I introduce details of the sample and the auxiliary radio and IR data. Section 3 presents the main results, and these are discussed further in Sect. 4. Section 5 presents the main conclusions of the paper with a suggestion for further work.

\section{The Ĝ sample and auxiliary data}

Griffith et al. (2015) have identified $\sim 100000$ resolved sources detected by WISE, and located above the galactic plane $(b>$ $10^{\circ}$ ). By avoiding the Milky Way and similarly dense regions of the sky such as the LMC, and by applying various colour criteria, Griffith et al. (2015) eliminate confounding objects such as galactic stars, diffuse nebular emission, and other stellar artefacts. The resulting cleaned catlogue of $\sim 31000$ extended red objects is therefore biased to include nearby galaxies that are prominent mid-IR sources with extreme colours. In addition, Griffith et al. (2015) apply the AGENT methodology (Wright et al. 2014b) to identify 93 sources that have mid-IR colours consistent with $\gamma>0.25$ where $\gamma$ is the fraction of starlight reemitted in the mid-IR as waste heat products, as modelled by the Wright et al. (2014b) AGENT analysis of the four WISE observing bands (3.4, 4.6, 12, and 22 microns).

I have cross-matched the $\hat{G}$ sample of 93 sources (see Griffith et al. 2015, Table 9) with the NRAO/VLA Sky Survey (NVSS) $1.4 \mathrm{GHz}(20 \mathrm{~cm})$ radio catalogue (Condon et al. 1998). I have restricted our study to the 92 sources that fall within the NVSS survey area and have measured redshifts. Since the sources are all resolved by WISE, they are mostly local systems with the median redshift being 0.028 . The highest redshift source in the sample has $z=0.14525$. I compare the $\lambda 20 \mathrm{~cm}$ radio emission with the WISE (band 4) 22 micron data since for local galaxies this band is dominated by continuum emission. The remaining WISE bands are more sensitive to polycyclic aromatic hydrocarbon (PAH) spectral features. Redshifts for the sources, together with IRAS 60 and 100 micron flux densities (where available), were extracted from a search of the NASA/IPAC Extragalactic Database (NED) and SIMBAD. The WISE magnitude system was converted to Jansky following Wright et al. (2010). The main data are presented in Table 1.

\section{Determining k-corrected values of $q$ for the Ĝ subsample}

Following Appleton et al. (2004) I define $q_{22}=$ $\log \left(S_{22 \mu} / S_{20 \mathrm{~cm}}\right) \quad$ where $S_{22 \mu}$ and $S_{20 \mathrm{~cm}}$ are the source flux densities measured by WISE and NVSS at wavelengths of $22 \mu \mathrm{m}$ (WISE band 4) and $20 \mathrm{~cm}$. Since the majority of the source samples are typically located in the local universe, the values of $q_{22}$ derived are relatively insensitive to any reasonable k-correction. Nevertheless, a k-correction has been applied to the analysis presented here to ensure consistency with other authors' results obtained for higher- $z$ samples. For the radio, I adopt a k-correction factor of $(1+z)^{+0.7}$ following Appleton et al. (2004). For the mid-IR corrections, and in particular $q_{22}$, I assume an M82-like SED as presented in Sturm et al. (2000). Over the limited redshift range associated with the $\hat{G}$ sample (22-26 $\mu \mathrm{m})$, the $\mathrm{k}$-correction is also well modelled by a power-law: $(1+z)^{-2.45}$.

Figure 1 presents a plot of the k-corrected $22 \mu$ mid-IR luminosity $\left(L_{22} \mu, \mathrm{W} / \mathrm{Hz}\right)$ against the $20 \mathrm{~cm}$ radio luminosity $\left(L_{20} \mathrm{~cm}, \mathrm{~W} / \mathrm{Hz}\right)$ for the $\mathrm{G}$ sample (red dots). The data clearly show a strong correlation between the mid-IR and radio luminsoities. A formal fit to the observed correlation yields

$\log \left(L_{22 \mu \mathrm{m}}\right)=(0.76 \pm 0.09) \log \left(L_{20} \mathrm{~cm}\right)+(6.65 \pm 0.37)$.

For a subset of the $\hat{G}$ sample (37 of the 92 sources) identified by Griffith et al. (2015) as galaxies (so excluding AGN, Sy 1 \&2, etc.) I similarly find

$\log \left(L_{22 \mu \mathrm{m}}\right)=(0.79 \pm 0.15) \log \left(L_{20 \mathrm{~cm}}\right)+(6.08 \pm 0.33)$.

In addition, a k-corrected value of $q_{22}=1.35 \pm 0.42$ is derived for the full sample. By comparison, a value of $q_{22}=1.40 \pm 0.34$ is also derived, again for the same subset of 37 sources identified as galaxies. Table 1 presents the values of $q$ derived for the full source sample, including upper limits for the ten sources that are below the $2.5 \mathrm{mJy}$ detection threshold of NVSS. The table is presented in order of decreasing $q_{22}$.

As far as I can ascertain, $q_{22}$ has not yet been derived for any other source samples observed by WISE. Appleton et al. (2004) derived a zero-redshift value for $q_{24}=0.84 \pm 0.28$ for a source sample derived from Spitzer observations of the First Look Survey (FLS). In Fig. 1 I also present the data for the $\hat{\mathrm{G}}$ sample together with a k-corrected plot of $L_{22} \mu$ vs. $L_{20} \mathrm{~cm}(\mathrm{~W} / \mathrm{Hz})$ for 124 galaxies located in the FLS that I also identify in the WISE all-sky catalogue with sources of known redshift, such that $z<0.2$ (Marleau et al. 2007). As expected, the FLS sample (plotted as triangles) clearly shows a strong correlation between the mid-IR and radio luminosities with a spread that is smaller than the $\hat{\mathrm{G}}$ galaxy sample. The slope of the best linear fit to the FLS data is similar to that seen in the $\hat{\mathrm{G}}$ subsample. A formal fit to the observed correlation in the FLS galaxy sample yields

$\log \left(L_{22 \mu \mathrm{m}}\right)=(0.70 \pm 0.07) \log \left(L_{20 \mathrm{~cm}}\right)+(7.36 \pm 0.22)$.

I derive a k-corrected $q_{22}=0.87 \pm 0.27$ for this FLS subsample. Considering the small wavelength difference, this is plainly consistent with the values of $q_{24}$ derived by Appleton et al. (2004).

Inspection by eye of Fig. 1, suggests that the mean difference between the $\hat{\mathrm{G}}$ and FLS samples $\left(q_{22}=1.35 \pm 0.42 \mathrm{vs}\right.$. $\left.q_{22}=0.87 \pm 0.27\right)$ may be significant. Indeed, applying Student's 


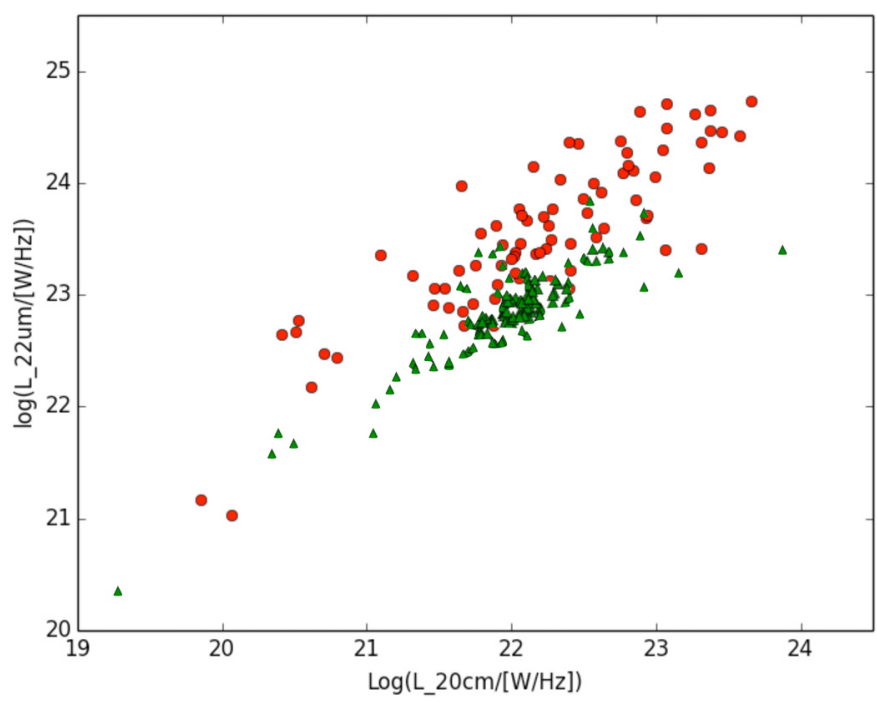

Fig. 1. k-corrected $22 \mu$ mid-IR luminosity $\left(L_{22} \mu\right.$, W/Hz), plotted against the $20 \mathrm{~cm}$ radio luminosity $\left(L_{20} \mathrm{~cm}, \mathrm{~W} / \mathrm{Hz}\right)$ for the $\hat{\mathrm{G}}$ (red circles) and FLS (green triangles) samples.

T-test (Student 1908) to the data shows the difference between the means $(0.48)$ to be statistically significant at the $95 \%$ confidence level (with the two-tailed $P$ value $<0.0001, t(204)=9.99$, a pooled variance of 0.45 , and a $95 \%$ confidence interval of the mean difference ranging between lower and upper limits of 0.3852 to 0.5748 ).

The value of $q_{\text {FIR }}$ was also determined for a subset of the $\hat{\mathrm{G}}$ sample (with both 60 and 100 micron flux densities) finding $q_{\text {FIR }}=2.45+/-0.39$. This is consistent with values found in much larger local galaxy samples, e.g. $q_{\mathrm{FIR}}=2.34 \pm 0.1$ (Yun et al. 2001).

Systematic underestimates of the radio flux density do not seem to be a major factor in our analysis, despite the extended nature of the sources, and the limited uv-coverage afforded by the NVSS snapshot observations. In particular, the fitted sizes of the sources are typically less than the NVSS synthesised beam (i.e. $<45$ arcsec). I conclude that the higher value of $q$ for the $\hat{\mathrm{G}}$ subsample is a physical characteristic of the source sample, and this has its origins in enhanced mid-IR emission rather than some systematic radio defecit.

Figure 2 presents a plot of $q_{22}$ against the $22 \mu$ mid-IR luminosity $\left(L_{22} \mu, \mathrm{W} / \mathrm{Hz}\right)$ of the $\hat{\mathrm{G}}$ sample. Outlying sources with $q_{22}>2$ are positioned above the dashed line. Details of the same sources are also presented towards the top of Table 1.

\section{Discussion}

Both the mid-IR and radio luminsoties of sources in the $\hat{G}$ subsample are strongly correlated with each other. This clearly demonstrates that the source sample as a whole follows the wellestablished mid-IR radio correlation associated with natural astrophysical processes such as massive star formation. One interesting, though perhaps not unexpected feature of the $\hat{G}$ sample is that the mean value of $q_{22}$ appears to be statistically different and indeed larger than that determined for the FLS sample. The fact that the values of $q_{\text {FIR }}$ are comparable between the samples suggests that this probably reflects a systematic excess in midIR emission associated with the $\hat{G}$ sample rather than a deficit of the sample in the radio domain.

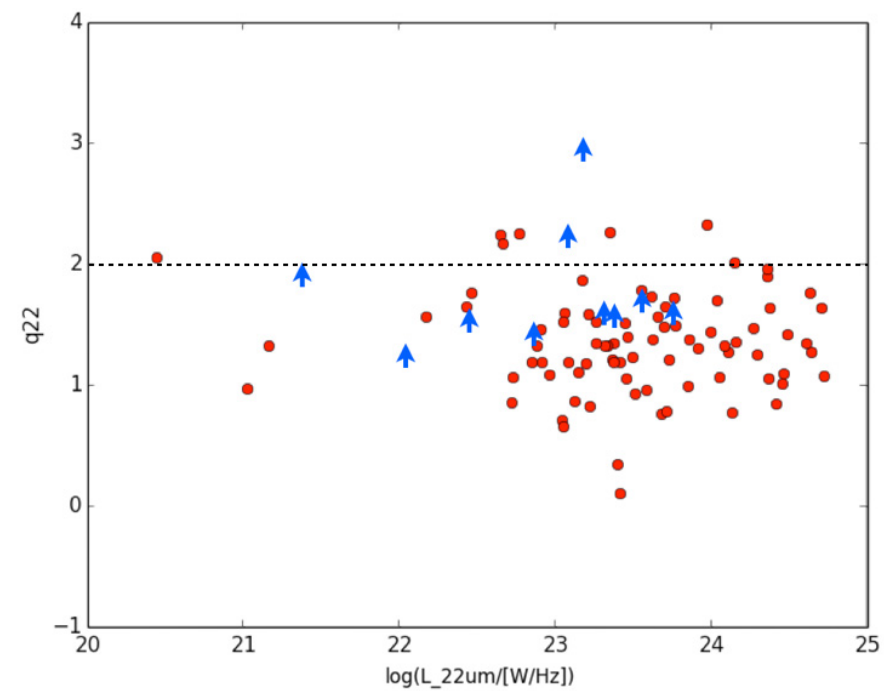

Fig. 2. k-corrected values of $q_{22}$ plotted against the $22 \mu$ mid-IR luminosity $\left(L_{22 \mu}, \mathrm{W} / \mathrm{Hz}\right)$ for the $\hat{\mathrm{G}}$ sample (red filled circles). Above the dashed line, lie the 9 outliers from the sample with $q_{22}>2$. Sources not detected in the NVSS radio survey show lower limits for $q_{22}$, and are presented as arrows.

High values of $q_{22}$ would be expected for systems dominated by Kardashev Type III civilisations, and this makes the outliers in the $\hat{G}$ sample of particular interest. Sources with $q_{22}>2$ (i.e. those lying $>1.5 \sigma$ from the $\hat{\mathrm{G}}$ sample mean or $>4 \sigma$ from the FLS sample mean) include MCG+02-60-017, IC 342, ESO 40028, NGC 814, NGC 4747, NGC 5253, UGC 3097, NGC 4355, and NGC 1377. Astrophysical explanations for high values of $q$ in the mid-IR include (i) very young star forming systems in which the synchrotron radio component is not fully established or (ii) obscured AGNs that heat nuclear dust to relatively warm temperatures. Of the sources with $q_{22}>2$ presented here, NGC 1377, NGC 4355, and IC 342 have been studied in some depth. Altao et al. (2012) favour an interpretation for NGC 1377 in which the prominent molecular outflow is driven by a young AGN embedded in a dust enshrouded nucleus. By comparison, IC 342 is a nearby face-on barred spiral galaxy with a central nuclear starburst, fed by bar-driven gas inflow (Schinnerer et al. 2003). The compact nucleus of NGC 4355 (also known as NGC 4418) also harbours an extremely rich and dusty molecular environment, but it is unclear whether a compact starburst or an AGN (or some combination of both) power the strong mid-IR compnent (Varenius et al. 2014). These three examples are probably typical representations of the range of types that dominate the $\hat{G}$ sample with large values of $q_{22}$.

\section{Conclusions and next steps}

In this paper, I have demonstrated that the IR-radio correlation can be employed as a useful diagnostic in distinguishing between mid-IR emission produced by natural astrophysical processes and that generated by artificial means, e.g. the waste heat energy associated with Type III civilisations. In particular, galaxies dominated by Type III civilisations should present themselves as extreme outliers to the mid-IR radio correlation with values of $q_{22}>2$. In this way, the mid-IR radio correlation can be used to eliminate false positives from the $\hat{G}$ sample, and to identify those systems that deserve further detailed study. 
The observations presented here demonstrate that the $\hat{\mathrm{G}}$ sample of 93 sources (Griffith et al. 2015, Table 9) typically follow the IR-radio correlation. I suggest that the vast majority, if not all of these sources present mid-IR emission associated with natural astrophysical processes. Those sources with $q_{22}>2$ that have not yet been widely studied in the literature deserve further investigation, however. Nevertheless, from the bulk properties of the $\hat{G}$ sample presented here, I conclude that these sources do not obviously harbour Karadashev Type III civilisations, and that therefore such civilisations are either extremely rare or do not exist in the local universe.

Finally, it should be noted that the IR-radio correlation is also known to hold on sub-galactic scales (e.g. Murphy 2006). A comparison of resolved mid-IR and radio images of nearby galaxies on kpc scales can also be useful in identifying artificial mid-IR emission from advanced civilisations that lie between the Types II and III. While Wright et al. (2014a) venture that Type III civilisations should emerge rapidly from Type IIs, it might be that some specific galactic localities are preferred (see e.g. Cirkovic \& Bradbury 2006) or are to be best avoided, e.g. the galactic centre. A comparison of the resolved radio and midIR structures can therefore also be relevant to future searches of waste heat associated with advanced civilisations.

Acknowledgements. Part of this work was supported by an IBM Faculty Award. This research has made use of the NASA/IPAC Extragalactic Database (NED) which is operated by the Jet Propulsion Laboratory, California Institute of Technology, under contract with National Aeronautics and Space Administration. This research has made use of the SIMBAD database, operated at CDS, Strasbourg, France. This research has made use of the NASA/IPAC Infrared Science Archive, which is operated by the Jet Propulsion Laboratory, California Institute of Technology, under contract with the National Aeronautics and Space Administration. I would like to thank the anonymous referee for very helpful and constructive comments, these helped to strengthen and improve the final version of this paper.

\section{References}

Altao, S., Muller, S., Sakamoto, K., et al. 2012, A\&A, 546, A68 Annis, J., 1999, J. British Interplanet. Soc., 52, 33

Appleton, P. N., Fadda, D. T., Marleau, F. R., et al. 2004, ApJS, 154, 147 Carilli, C. L., \& Yun, M. S. 1999, ApJ, 513, 13

Carrigan, R. A. 2009, ApJ, 698, 2075

Cirkovic, M. M., \& Bradbury, R. J. 2006, New Astron., 11, 628

Condon, J. J. 1992, ARA\&A, 30, 575

Condon, J. J., Cotton, W. D., Greisen, E. W., et al. 1998, AJ, 115, 1693

Dyson, F. J. 1960, Science, 131, 1667

Elbaz, D., Cesarsky, C. J., Chanial, P., et al. 2002, A\&A 384, 848

Garrett, M. A. 2002, A\&A, 384, 19

Griffith, R. L., Wright, J. T., Maldonado, J., et al. 2015, ApJS, 217, 25

Helou, G., Soifer, B. T., \& Rowan-Robinson, M. 1985, ApJ, 298, L7

Ivison, R. J., Greve, T. R, Smail, I., et al. 2002, MNRAS, 337, 1

Kardashev, N. S. 1964, Sov. Astron., 8, 217

Marleau, F. R, Fadda, D., Appleton, P. N., et al. 2007, ApJ, 663, 218

Murphy, E. J., Braun, R., Helou, G., et al., 2006, ApJ, 638, 157

Roy A. L., Norris R. P., Kesteven M. J., Troup E. R., \& Reynolds J. E., 1998, MNRAS, 301, 1019

Schinnerer, E., Boker, T., \& Meier, D. S. 2003, ApJ, 591, L115

Student. 2008, Biometrika, 6, 1

Sturm, E., Lutz, D., Tran, D., et al. 2000, A\&A, 358, 481

van der Kruit, P. C. 1971, A\&A, 15, 110

Varenius, E., Conway, J. E., Martí-Vidal, I., et al. 2014, A\&A, 566, A15

Wright, E. L., Eisenhardt, P. R. M., Mainzer, A. K., et al. 2010, AJ, 140, 1868

Wright, J. T., Mullan, B. A., Sigurđsson, S., \& Povich, M. S. 2014a, ApJ, 792, 26

Wright, J. T., Griffith, R. L., Sigurđsson, S., Povich, M. S., \& Mullan, B. A. 2014b, ApJ, 792, 27

Yun, M. S., Reddy, N. A., \& Condon, J. J. 2001, ApJ, 554, 803

Pages 5 to 6 are available in the electronic edition of the journal at http: //www . aanda. org 
M. A. Garrett: Application of the mid-IR radio correlation to the $\hat{G}$ sample

Table 1. The Ĝ sample studied in this paper, including values (or upper limints) of $q_{22}$.

\begin{tabular}{|c|c|c|c|c|c|c|c|c|}
\hline RA (J2000) & Dec (J2000) & $S_{20 \mathrm{~cm}}$ & WISE-4 (mag) & $q$ & $\mathrm{z}$ & $\gamma$ & SIMBAD Type & Name \\
\hline 033639.05 & -205406.8 & $<2.5$ & 1.5 & $>2.88$ & 0.005921 & 0.5 & $\mathrm{GiG}$ & NGC 1377 \\
\hline 122654.61 & -005239.1 & 40.8 & -0.15 & 2.326 & 0.007048 & 0.85 & Sy2 & NGC 4355 \\
\hline 043548.45 & +021529.6 & 3.9 & 2.54 & 2.263 & 0.012014 & 0.52 & $\mathrm{G}$ & UGC 3097 \\
\hline 133955.96 & -313824.4 & 84.7 & -0.73 & 2.248 & 0.001345 & 0.60 & AGN & NGC 5253 \\
\hline 125145.54 & +254628.5 & 7.4 & 1.92 & 2.244 & 0.003966 & 0.53 & IG & NGC 4747 \\
\hline 021037.63 & -154624.2 & 5.0 & 2.54 & 2.164 & 0.005405 & 0.54 & G & NGC 814 \\
\hline 202825.49 & -330420.5 & $<2.5$ & 3.29 & $>2.156$ & 0.01228 & 0.32 & G & ESO $400-28$ \\
\hline 034648.35 & +680546.5 & 190.7 & -1.12 & 2.054 & $7.7 \mathrm{e}-05$ & 0.49 & GiG & IC 342 \\
\hline 234709.20 & +153548.3 & 9.2 & 2.2 & 2.008 & 0.026105 & 0.65 & Sy2 & $\mathrm{MCG}+02-60-017$ \\
\hline 003652.44 & -333316.8 & 26.8 & 1.17 & 1.963 & 0.020558 & 0.66 & AGN & ESO $350-38$ \\
\hline 225234.71 & +244349.4 & 7.3 & 2.67 & 1.9 & 0.04193 & 0.60 & AGN & Mrk 309 \\
\hline 181603.19 & +473705.4 & 3.2 & 3.74 & 1.862 & 0.01706 & 0.39 & G & 2MASX J18160312+4737056 \\
\hline 102508.18 & +170914.1 & $<2.5$ & 4.14 & $>1.829$ & 0.002512 & 0.58 & IG & NGC 3239 \\
\hline 100125.94 & +154612.2 & 44.0 & 1.14 & 1.776 & 0.007895 & 0.34 & G & NGC 3094 \\
\hline 055542.61 & +032331.8 & 32.5 & 1.52 & 1.763 & 0.002669 & 0.72 & $\mathrm{H} 2 \mathrm{G}$ & UGCA 116 \\
\hline 043400.03 & -083444.9 & 137.1 & -0.07 & 1.756 & 0.015851 & 0.63 & AGN & NGC 1614 \\
\hline 103833.62 & -071014.4 & 66.6 & 0.8 & 1.733 & 0.007283 & 0.61 & G & IC 630 \\
\hline 054323.63 & +540044.2 & 6.9 & 3.24 & 1.715 & 0.027019 & 0.51 & G & 2MASX J05432362+5400439 \\
\hline 130220.39 & -154559.0 & 34.8 & 1.55 & 1.702 & 0.016745 & 0.53 & $\mathrm{GiG}$ & MCG-02-33-099 X \\
\hline 051646.24 & -122059.4 & 6.9 & 3.47 & 1.651 & 0.00636 & 0.26 & $\mathrm{G}$ & 6dFGS gJ051646.2-122100 \\
\hline 083538.40 & -011407.1 & 2.7 & 4.38 & 1.645 & 0.0438 & 0.36 & G & 2MASX J08353838-0114072 \\
\hline 173801.51 & +561325.9 & 5.8 & 3.51 & 1.634 & 0.06519 & 0.45 & Sy2 & 2MASX J17380143+5613257 \\
\hline 205724.32 & +170738.5 & 43.2 & 1.43 & 1.632 & 0.035 & 0.70 & G & IRAS F20550+1655SE \\
\hline 173431.80 & +471301.7 & $<2.5$ & 4.54 & $>1.621$ & 0.03901 & 0.29 & G & 2MASX J17343177+4713010 \\
\hline 154813.36 & -245309.6 & 6.9 & 3.59 & 1.593 & 0.0139 & 0.39 & EmG & ESO 515-7 \\
\hline 194605.40 & +640850.1 & 5.5 & 3.84 & 1.585 & 0.018786 & 0.26 & G & 2MASX J19460544+6408494 \\
\hline 055652.46 & -052303.8 & 3.1 & 4.55 & 1.564 & 0.007749 & 0.26 & LSB & 2MASXI J0556522-052308 \\
\hline 233614.11 & +020917.9 & 65.8 & 1.24 & 1.559 & 0.00935 & 0.49 & AGN & NGC 7714 \\
\hline 105416.74 & -394019.3 & 5.6 & 3.98 & 1.524 & 0.016611 & 0.31 & G & ESO 318-23 \\
\hline 142837.03 & -394844.1 & $<2.5$ & 4.81 & $>1.521$ & 0.03298 & 0.28 & IG & ESO $326-24$ \\
\hline 182552.75 & +375241.6 & $<2.5$ & 4.74 & $>1.52$ & 0.05492 & 0.32 & IR & IRAS $18241+3750$ \\
\hline 120913.87 & +265237.4 & 2.1 & 5.0 & 1.518 & 0.03447 & 0.26 & G & LEDA 38612 \\
\hline 064339.31 & -271217.6 & 7.0 & 3.74 & 1.514 & 0.02353 & 0.27 & GiG & 2MASX J06433935-2712180 \\
\hline 024506.40 & -020727.7 & $<2.5$ & 4.86 & $>1.496$ & 0.03656 & 0.29 & G & 2MFGC 2186 \\
\hline 134547.40 & +700445.9 & 8.3 & 3.58 & 1.493 & 0.032012 & 0.37 & G & 2MASX J13454733+7004455 \\
\hline 195751.88 & -322128.2 & 13.0 & 3.15 & 1.48 & 0.0239 & 0.35 & EmG & 6dFGS gJ195751.9-322128 \\
\hline 005404.02 & +730505.7 & 113.8 & 0.84 & 1.473 & 0.015823 & 0.54 & G & MCG+12-02-001 \\
\hline 050147.35 & -181000.8 & $<2.5$ & 5.02 & $>1.463$ & 0.01319 & 0.30 & G & NGC 1739 \\
\hline 064540.95 & +433407.5 & 3.3 & 4.72 & 1.453 & 0.01982 & 0.27 & G & 2MASX J06454097+4334069 \\
\hline 132021.98 & -233225.9 & 8.1 & 3.71 & 1.435 & 0.0448 & 0.35 & EmG & 2MASX J13202200-2332256 \\
\hline 224458.08 & -014600.4 & 13.3 & 3.16 & 1.417 & 0.06262 & 0.41 & G & 2MASX J22445816-0145589 \\
\hline 131503.51 & +243707.8 & 30.8 & 2.45 & 1.4 & 0.013049 & 0.61 & $\mathrm{Q}$ ? & IC 860 \\
\hline 024143.24 & +454626.6 & 7.5 & 3.99 & 1.372 & 0.032736 & 0.38 & $\mathrm{G}$ & 2MASX J02414325+4546272 \\
\hline 194112.82 & +630542.9 & 5.0 & 4.37 & 1.37 & 0.05267 & 0.31 & G & 2MASX J19411289+6305430 \\
\hline 185222.44 & -293620.7 & 16.0 & 3.18 & 1.354 & 0.042355 & 0.37 & EmG & 6dFGS gJ185222.4-293621 \\
\hline 191227.31 & -290235.7 & 7.3 & 4.1 & 1.349 & 0.025629 & 0.35 & EmG & ESO $459-7$ \\
\hline 151806.13 & +424444.8 & 50.3 & 1.96 & 1.347 & 0.0405 & 0.60 & LIN & IRAS F15163+4255NW \\
\hline 004250.05 & -365243.2 & 6.7 & 4.22 & 1.34 & 0.02374 & 0.30 & G & 6dFGS gJ004250.1-365241 \\
\hline 063226.08 & -243208.3 & $<2.5$ & 5.29 & $>1.34$ & 0.02416 & 0.30 & G & ESO 490-11 \\
\hline 152309.66 & -393448.2 & 7.2 & 4.17 & 1.327 & 0.02541 & 0.34 & $\mathrm{G}$ & 2MASX J15230967-3934481 \\
\hline 102050.93 & -171859.4 & 5.2 & 4.52 & 1.323 & 0.0293 & 0.32 & EmG & MCG-03-27-005 \\
\hline 030445.12 & +074739.2 & 2.3 & 5.42 & 1.321 & 0.02682 & 0.27 & $\mathrm{G}$ & 2MASX J03044511+0747394 \\
\hline 121539.36 & +361935.1 & 33.7 & 2.59 & 1.321 & 0.000977 & 0.59 & SBG & NGC 4228 \\
\hline 020805.42 & -291432.7 & 6.4 & 4.19 & 1.321 & 0.0636 & 0.41 & G & 6dFGS gJ020805.4-291433 \\
\hline 025559.96 & +4748 19.3 & 19.0 & 3.16 & 1.302 & 0.031332 & 0.37 & G & MCG+08-06-022 \\
\hline 001850.88 & -102236.6 & 42.4 & 2.37 & 1.275 & 0.027 & 0.52 & EmG & MCG-02-01-051 \\
\hline 153457.25 & +233011.5 & 326.3 & 0.19 & 1.272 & 0.018116 & 0.79 & SyG & Arp 220 \\
\hline 150253.22 & +165508.4 & 18.7 & 3.4 & 1.226 & 0.021223 & 0.33 & rG & NVSS J150253+165507 \\
\hline 012002.63 & +142142.5 & 49.8 & 2.24 & 1.251 & 0.031555 & 0.49 & LIN & MCG+02-04-025 \\
\hline 000820.57 & +403755.9 & 6.9 & 4.44 & 1.211 & 0.046148 & 0.54 & Sy2 & 2MASX J00082041+4037560 \\
\hline 092338.22 & -251634.9 & 14.4 & 3.74 & 1.203 & 0.0214 & 0.32 & GiP & 6dFGS gJ092338.2-251635 \\
\hline
\end{tabular}


Table 1. continued.

\begin{tabular}{|c|c|c|c|c|c|c|c|c|}
\hline RA (J2000) & $\operatorname{Dec}(\mathrm{J} 2000)$ & $S_{20 \mathrm{~cm}}$ & WISE-4 (mag) & $q$ & $\mathrm{z}$ & $\gamma$ & SIMBAD Type & Name \\
\hline 042851.45 & +6934 47.1 & 9.3 & 4.26 & 1.192 & 0.016168 & 0.33 & G & 2MASX J04285125+6934469 \\
\hline 044738.41 & -172601.8 & 11.7 & 4.02 & 1.192 & 0.013299 & 0.39 & G & ESO 552-5 \\
\hline 140736.99 & +160121.6 & 4.7 & 4.98 & 1.185 & 0.02768 & 0.25 & G & 2MASX J14073693+1601212 \\
\hline 093548.86 & -291955.6 & 4.1 & 5.08 & 1.185 & 0.0431 & 0.32 & EmG & ESO 434-13 \\
\hline 125324.16 & -234545.6 & 3.1 & 5.37 & 1.184 & 0.0475 & 0.31 & EmG & 6dFGS gJ125324.2-234546 \\
\hline 181041.37 & +250723.2 & 9.7 & 4.23 & 1.178 & 0.02213 & 0.38 & G & 2MASX J18104135+2507238 \\
\hline 054738.65 & -103552.8 & $<2.5$ & 5.76 & $>1.169$ & 0.01154 & 0.25 & G & 6dFGS gJ054738.7-103552 \\
\hline 054323.63 & +540044.2 & 6.9 & 4.77 & 1.103 & 0.027019 & 0.56 & G & 2MASX J05432362+5400439 \\
\hline 105918.14 & +243234.6 & 57.0 & 2.44 & 1.098 & 0.042876 & 0.50 & LIN & 2XMM J105918.1+243234 \\
\hline 093930.15 & +062613.0 & 5.7 & 5.04 & 1.082 & 0.02459 & 0.25 & $\mathrm{rG}$ & NVSS J093929+062610?2MAS \\
\hline 134442.10 & +555313.3 & 144.7 & 1.51 & 1.072 & 0.03734 & 0.55 & Sy2 & Mrk 273 \\
\hline 045510.80 & +053512.6 & 7.5 & 4.81 & 1.065 & 0.01671 & 0.38 & G & 2MASX J04551070+0535126 \\
\hline 232610.59 & -303106.1 & 10.5 & 4.29 & 1.065 & 0.0641 & 0.28 & EmG & 6dFGS gJ232610.6-303106 \\
\hline 163600.57 & +101340.4 & 4.1 & 5.09 & 1.055 & 0.14525 & 0.43 & $\mathrm{G}$ & 2MASX J16360060+1013396 \\
\hline 150029.00 & -262649.2 & 37.8 & 3.08 & 1.053 & 0.01751 & 0.65 & $\mathrm{H} 2 \mathrm{G}$ & 2MASX J15002897-2626487 \\
\hline 121346.00 & +024840.3 & 23.3 & 3.54 & 1.008 & 0.0731 & 0.55 & LIN & LEDA 39024 \\
\hline 225451.03 & +374220.8 & 3.8 & 5.49 & 0.993 & 0.091137 & 0.28 & $\mathrm{G}$ & 2MASX J22545096+3742205 \\
\hline 025941.29 & +251415.0 & 33.1 & 3.49 & 0.969 & 0.001265 & 0.54 & G & NGC 1156 \\
\hline 011607.20 & +3305 21.7 & 75.4 & 2.57 & 0.959 & 0.016044 & 0.54 & Sy2 & NGC 449 \\
\hline 054421.56 & -135311.9 & 10.5 & 4.7 & 0.932 & 0.0403 & 0.31 & $\mathrm{G}$ & 2MASX J05442151-1353116 \\
\hline 173429.01 & -040541.7 & 6.2 & 5.44 & 0.87 & 0.0363 & 0.35 & G & 6dFGS gJ173428.9-040542 \\
\hline 051521.42 & -262817.1 & 20.6 & 4.25 & 0.855 & 0.012809 & 0.30 & EmG & ESO $486-39$ \\
\hline 124930.16 & -112403.4 & 73.4 & 2.79 & 0.842 & 0.047826 & 0.59 & Sy2 & IRAS 12468-1107 \\
\hline 161833.99 & +132425.9 & 4.6 & 5.85 & 0.818 & 0.04953 & 0.28 & $\mathrm{G}$ & 2MASX J16183392+1324253 \\
\hline 222149.97 & +395024.0 & 7.8 & 5.31 & 0.779 & 0.069725 & 0.29 & G & 2MASX J22215143+3950240 \\
\hline 003443.48 & -000226.6 & 57.0 & 3.25 & 0.774 & 0.042529 & 0.54 & Sy2 & 2MFGC 403 \\
\hline 025609.76 & -153943.5 & 10.9 & 5.04 & 0.756 & 0.05877 & 0.33 & $\mathrm{G}$ & 6dFGS gJ025609.8-153946 \\
\hline 112402.72 & -282315.4 & 52.1 & 3.6 & 0.711 & 0.01374 & 0.43 & Sy2 & IRAS 11215-2806 \\
\hline 114543.59 & -114712.6 & 33.3 & 4.21 & 0.655 & 0.018463 & 0.29 & EmG & 6dFGS gJ114543.6-114712 \\
\hline 105052.15 & +010944.2 & 32.7 & 4.94 & 0.343 & 0.03952 & 0.29 & GiG & IC $649 \mathrm{~S}$ \\
\hline 130842.02 & -242257.8 & 474.5 & 2.72 & 0.103 & 0.014 & 0.50 & Sy2 & PKS 1306-241 \\
\hline
\end{tabular}

